\title{
The mixed chemistry phenomenon in Galactic Bulge PNe $\mathrm{Pe}^{\star \star \star}$
}

\author{
J. V. Perea-Calderón ${ }^{1}$, D. A. García-Hernández ${ }^{2}$, P. García-Lario ${ }^{3}$, R. Szczerba ${ }^{4}$, and M. Bobrowsky ${ }^{5}$ \\ ${ }^{1}$ European Space Astronomy Centre, INSA SA, PO Box 78, 28080 Madrid, Spain \\ e-mail: Jose.Perea@sciops.esa.int \\ 2 Instituto de Astrofísica de Canarias, C/ via Láctea s/n, 38200 La Laguna, Spain \\ 3 Herschel Science Centre. European Space Astronomy Centre, Research and Scientific Support Department of ESA, \\ Villafranca del Castillo, PO Box 78, 28080 Madrid, Spain \\ 4 N. Copernicus Astronomical Center, Rabiańska 8, 87-100 Toruń, Poland \\ 5 Department of Physics. University of Maryland, College Park, MD 20742-4111, USA
}

Received 2 December 2008 / Accepted 13 January 2009

\section{ABSTRACT}

\begin{abstract}
Aims. We investigate the dual-dust chemistry phenomenon in planetary nebulae ( $\mathrm{PNe})$ and discuss reasons for its occurrence, by analyzing Spitzer/IRS spectra of a sample of 40 Galactic PNe among which 26 belong to the Galactic Bulge (GB).

Methods. The mixed chemistry is derived from the simultaneous detection of Polycyclic Aromatic Hydrocarbon (PAH) features in the 6-14 $\mu \mathrm{m}$ range and crystalline silicates beyond $20 \mu \mathrm{m}$ in the Spitzer/IRS spectra.

Results. Out of the 26 planetary nebulae observed in the Galactic Bulge, 21 show signatures of dual-dust chemistry. Our observations reveal that the simultaneous presence of oxygen and carbon-rich dust features in the infrared spectra of [WC]-type planetary nebulae is not restricted to late/cool [WC]-type stars, as previously suggested in the literature, but is a common feature associated with all [WC]-type planetary nebulae. Surprisingly, we found that the dual-dust chemistry is seen also in all observed weak emission-line stars (wels), as well as in other planetary nebulae with central stars being neither [WC] nor wels. Most sources observed display crystalline silicate features in their spectra, with only a few PNe exhibiting, in addition, amorphous silicate bands.

Conclusions. We appear to detect a recent change of chemistry at the end of the Asymptotic Giant Branch (AGB) evolution in the lowmass, high-metallicity population of GB PNe observed. The deficit of C-rich AGB stars in this environment suggests that the process of PAH formation in PNe occurs at the very end of the AGB phase. In addition, the population of low-mass, O-rich AGB stars in the Galactic Bulge, do not exhibit crystalline silicate features in their spectra. Thus, the high detection rate of dual-dust chemistry that we find cannot be explained by long-lived O-rich (primordial or circumbinary) disks. Our most plausible scenario is a final thermal pulse on the AGB (or just after), which could produce enhanced mass loss, capable of removing/mixing (sometimes completely) the remaining H-rich envelope and exposing the internal C-rich layers, and generating shocks responsible for the silicate crystallization.
\end{abstract}

Key words. planetary nebulae: general - circumstellar matter - stars: Wolf-Rayet

\section{Introduction}

Planetary Nebulae (PNe) are the result of the evolution of lowand intermediate-mass stars $\left(1-8 M_{\odot}\right)$, where the circumstellar gas previously expelled during the Asymptotic Giant Branch (AGB) phase is ionized by the central star (CS). The infrared emission from dusty circumstellar shell that developes at the end of the AGB can remain strong during the PN phase, and thus be easily detectable with infrared telescopes.

An interesting class of PNe are those with C-rich Wolf-Rayet ([WC]-type) central stars (the so-called "WCPNe"), which have little or no $\mathrm{H}$ in their atmospheres. Observations completed by the Infrared Space Observatory (ISO) suggest that the shells around some WCPNe exhibit a characteristic dual-dust (or mixed) chemistry (Waters et al. 1998a,b; Cohen et al. 1999, 2002), which is not yet well understood. This is deduced from the simultaneous presence of the well-known family of infrared bands at 3.3, 6.2, “7.7”, 8.6, and $11.3 \mu \mathrm{m}$ due to Policyclic

\footnotetext{
* Based on observations made with the Spitzer Space Telescope, which is operated by the Jet Propulsion Laboratory, California Institute of Technology, under NASA contract 1407.

$\star \star$ Tables A1, A2 and Figs. B1 to B6 are only available in electronic form at http://www. aanda.org
}

Aromatic Hydrocarbons (PAHs: C-rich) (Leger \& Puget 1984; Allamandola et al. 1985), and several relatively broad bands (the strongest at $23.5,27.5$, and $33.8 \mu \mathrm{m}$ ) attributed to crystalline silicates (O-rich), such as olivines and pyroxenes (Waters et al. 1998a) in the ISO spectra. The mixed chemistry phenomenon appears to be common among late-type/cool WCPNe, while only one (NGC 5315) out of 6 early-type/hot WCPNe observed with ISO shows this dual-dust chemistry. In total about $50 \%$ of the WCPNe show mixed chemistry (Cohen et al. 2002; De Marco \& Soker 2002). Note, however, that PAH features seem to be present in most of the 16 WCPNe analyzed by Szczerba et al. (2001), with the clear exception of K2-16. Therefore, we should keep in mind that, due to the poor quality of ISO band 4 data, the true percentage of WCPNe with mixed chemistry may be underestimated.

In this Letter, we report the results of a systematic, spectroscopic analysis completed with the Infrared Spectrograph (IRS, Houck et al. 2004) onboard the Spitzer Space Telescope (Werner et al. 2004) of a sample of 26 Galactic Bulge (GB) PNe around central stars, which are classified as late-type/cool [WC], earlytype/hot [WC], weak emission-line stars (wels) (Tylenda et al. 1993), and neither [WC] nor wels. We also briefly discuss the remaining 14 non-GB PNe in our sample. Section 2 describes 
Table 1. The sample of 21 Galactic Bulge PNe with dual-dust chemistry observed with Spitzer/IRS (program \#3633).

\begin{tabular}{|c|c|c|c|c|c|c|c|}
\hline IRAS name & PN name & $\begin{array}{c}\text { RA } \\
\text { J2000.0 }\end{array}$ & $\begin{array}{c}\text { Dec } \\
\text { J2000.0 }\end{array}$ & Spectral type ${ }^{a}$ & $\begin{array}{l}\text { Optical diameter }{ }^{d} \\
(\operatorname{arcsec})\end{array}$ & $\begin{array}{c}\text { Distance from GC } \\
\left(^{\circ}\right)\end{array}$ & Dust type \\
\hline 17480-3023 & M 3-44 & 175118.7 & -302353 & {$[\mathrm{WC} 11]^{b}$} & 4.4 & 1.91 & mixed \\
\hline 17549-3347 & H $1-43$ & 175814.5 & -334737 & {$[\mathrm{WC} 11]^{b}$} & 2.0 & 5.56 & mixed \\
\hline $17434-3307$ & M 1-27 & 174645.5 & -330835 & {$[\mathrm{WC} 11]^{b}$} & 5.3 & 4.21 & mixed \\
\hline $18061-2505$ & $\mathrm{MaC} 1-10$ & 180912.4 & -250435 & {$[\mathrm{WC} 8]^{b}$} & 10.0 & 6.52 & mixed \\
\hline $17355-2206$ & M 1-25 & 173830.3 & -220839 & [WC6 $^{b}$ & 4.6 & 6.99 & mixed \\
\hline $17425-2056$ & M 3-15 & 174531.8 & -205806 & {$[\mathrm{WC} 5]^{b}$} & 4.2 & 7.97 & mixed \\
\hline $17388-2440$ & $\mathrm{Hb} 4$ & 174153.1 & -244203 & {$[\mathrm{WC} 4]^{b}$} & 6.2 & 4.32 & mixed \\
\hline $18259-3132$ & Cn $1-5$ & 182911.7 & -312959 & {$[\mathrm{WC} 4]^{b}$} & 7.0 & 9.75 & mixed \\
\hline $17389-2409$ & M 2-14 & 174157.7 & -241111 & wels $^{b}$ & 0.0 & 4.82 & mixed \\
\hline $17496-2221$ & M 1-31 & 175241.4 & -222157 & wels $^{b}$ & 0.0 & 6.76 & mixed \\
\hline $18006-3117$ & M 2-27 & 180352.6 & -311747 & wels $^{b}$ & 4.8 & 4.60 & mixed \\
\hline $18054-2217$ & M 1-40 & 180826.0 & -221653 & wels $^{b}$ & 5.0 & 8.40 & mixed \\
\hline $18094-2450$ & H $1-61$ & 181234.0 & -245001 & wels $s^{b}$ & 0.0 & 7.27 & mixed \\
\hline $17156-3135$ & Th 3-4 & 171851.9 & -313906 & none $^{b}$ & 0.0 & 6.38 & mixed \\
\hline $17178-2900$ & M 3-38 & 172104.3 & -290260 & none $e^{c}$ & 0.0 & 5.37 & mixed \\
\hline $17217-2803$ & M 3-8 & 172452.2 & -280554 & none $e^{b}$ & 5.4 & 4.64 & mixed $^{e}$ \\
\hline $17262-2623$ & H $1-16$ & 172923.4 & -262605 & none $^{b}$ & $<5.0$ & 4.37 & mixed \\
\hline $17521-2144$ & $\mathrm{Hb} 6$ & 175507.1 & -214441 & none $^{b}$ & 5.0 & 7.50 & mixed \\
\hline $17523-3033$ & H $1-40$ & 175536.0 & -303332 & none $^{b}$ & 3.8 & 2.71 & mixed \\
\hline $18006-3241$ & H $1-50$ & 180353.5 & -324142 & none $^{b}$ & $<10$ & 5.43 & mixed \\
\hline $18100-3220$ & H $1-62$ & 181317.9 & -321942 & unknown & 0.0 & 6.85 & mixed \\
\hline
\end{tabular}

${ }^{a}$ None - neither [WC] nor wels; ${ }^{b}$ Górny et al. (2004); ${ }^{c}$ Górny et al. (2008); ${ }^{d}$ Strasbourg-ESO Catalogue of Galactic Planetary Nebulae, Acker et al. (1992), $0.0-\mathrm{PN}$ is a point-like source; ${ }^{e} \mathrm{PAHs}$ presence is tentative.

the infrared observations carried out and the data reduction performed, while the results obtained are presented in Sect. 3. Finally, in Sect. 4 we discuss our findings in the context of stellar evolution in the Galactic Bulge.

\section{Observations and data reduction}

The spectral data were taken with Spitzer/IRS under a General Observer program (\#3633, PI, Bobrowsky). We obtained Spitzer/IRS spectra of a sample of $40 \mathrm{PNe}^{1}$ in the $5.2-37.2 \mu \mathrm{m}$ range by using the Short-Low (SL: $5.2-14.5 \mu \mathrm{m} ; 64<R<128$ ), Short-High (SH: 9.9-19.6 $\mu \mathrm{m} ; R \sim 600$ ) and Long-High (LH: $18.7-37.2 \mu \mathrm{m} ; R \sim 600$ ) modules. All sources in our sample are bright at mid-infrared wavelengths, and it was easy to achieve a signal-to-noise ratio higher than 50 in each of the three modules $\mathrm{SL}, \mathrm{SH}$, and LH by using two exposure cycles of $6 \mathrm{~s}$. The starting point for our interactive data reduction were the coadded $2 \mathrm{D}$ flatfielded images (one for each nod position; pipeline version 12.3), in which "rogue" pixels were cleaned. For SL, the two nod positions were subtracted in order to remove the sky background. However, for the high-resolution modules no background subtraction was done since no sky measurements were taken and the SH and LH slits were too small for on-slit background subtraction. The spectra for each nod position were extracted from the $2 \mathrm{D}$ images, and wavelength and flux calibrated using the Spitzer IRS Custom Extractor (SPICE) with a point-source aperture. The 1D spectra were cleaned for bad data points, spurious jumps and glitches, combined and merged into one single spectrum per module for each target using SMART ${ }^{2}$ (Higdon et al. 2004). Finally, when required we scaled the LH spectra to the available Midcourse Space Experiment (MSX; Egan et al. 2003)

\footnotetext{
${ }^{1}$ Plots of all spectra are available online. Table 1 and online Tables A.1 and A.2 collect relevant information for our sample of PNe.

2 SMART was developed by the IRS Team at Cornell University and is available through the Spitzer Science Center at Caltech.
}

photometry at $21.34 \mu \mathrm{m}$, and the corresponding SL and SH spectra were also scaled upward to match the LH spectra. This is because some of the PNe studied here are extended sources (optical diameter $>4^{\prime \prime}$ ), giving a flux loss at the smaller SL and SH apertures. The MSX photometry at $21.34 \mu \mathrm{m}$ was selected as a reference point for flux calibration because this filter is less affected by nebular emission lines and dust emission features.

\section{Results}

In Table 1 , we present the names (Cols. 1 \& 2), coordinates (Cols. 3 \& 4), spectral type (Col. 5), optical diameter (Col. 6), distance from Galactic Center (GC: Col. 7), and dust type (Col. 8) for the $21 \mathrm{PNe}$ in our sample, which belong to the Galactic Bulge $\mathrm{e}^{3}$ and show dual-dust chemistry. The total number of GB PNe observed by us is 26 , so the occurrence of dual-dust chemistry (21/26) is much higher than the 6/11 observed in the sample of GB PNe discussed by Gutenkunst et al. (2008).

Figure 1 shows representative Spitzer/IRS spectra corresponding to 4 classes of PN CSs from Table 1: late-type/cool [WC] (H 1-43); early-type/hot [WC] (Cn 1-5); wels (M 1-40); and neither [WC] nor wels (H 1-16). In Appendix B, we present spectra for all 21 PNe from Table 1 (see Figs. B.1, B.2 and B.3), and for the remaining $5 \mathrm{~GB}$ PNe in our sample (Table A.1 in Appendix A contains relevant information for these sources), which show only the presence of silicates (Fig. B.4). The spectral type of the CS is shown below the PN name and "none" is used instead of "neither [WC] nor wels". All spectra exhibit a strong infrared continuum emission, which peaks between 20 and $40 \mu \mathrm{m}$, as observed in many other PNe detected by ISO. This strong infrared excess is produced by the thermal emission of dust still present in their remnant circumstellar AGB shells. Overimposed on this infrared continuum we can clearly distinguish the presence of narrow nebular emission lines.

\footnotetext{
3 A PN was counted as a member of the GB when its angular distance from the $\mathrm{GC}$ was $<10^{\circ}$ and its optical angular diameter was $<10^{\prime \prime}$.
} 


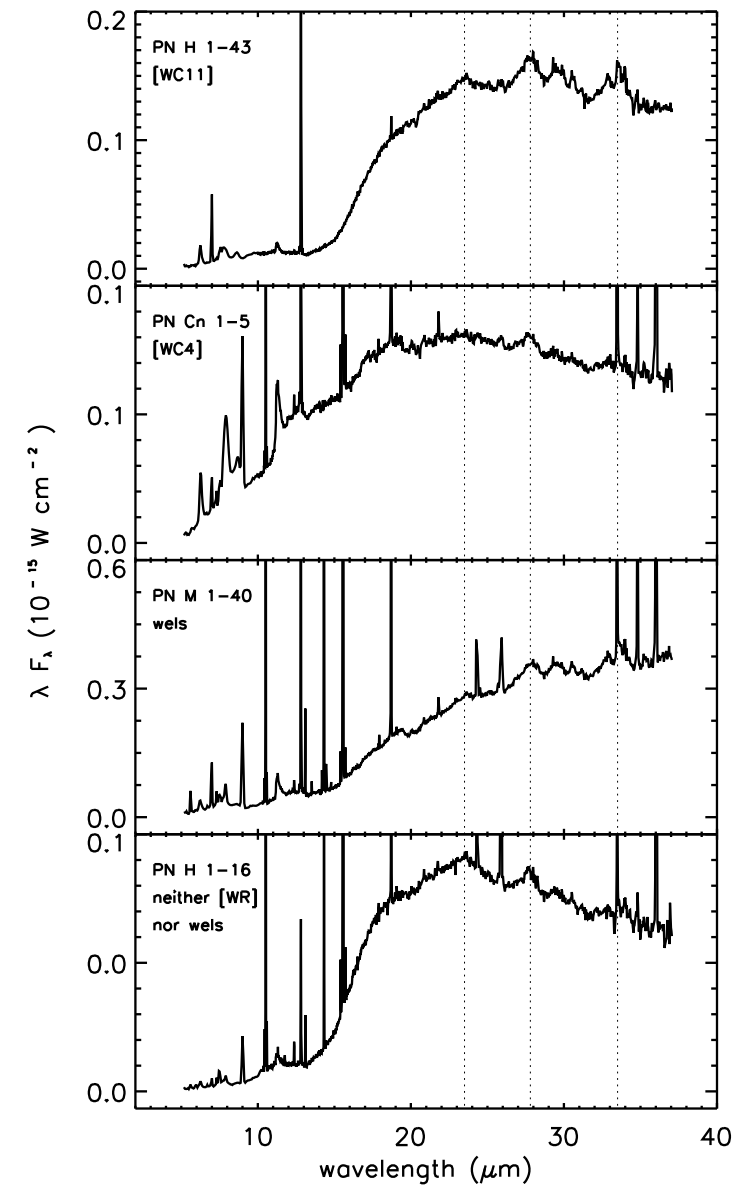

Fig. 1. Spitzer/IRS spectra of four representative GB PNe from our sample (from top to bottom: late-type/cool [WC], early-type/hot [WC], wels and neither [WC] nor wels). Note the simultaneous detection of PAH emission features in the 6-14 $\mu \mathrm{m}$ range (see Fig. 2) together with prominent crystalline silicate emission features (marked with dotted vertical lines at $23.5,27.5$ and $33.8 \mu \mathrm{m}$ ) beyond $20 \mu \mathrm{m}$, in all cases.

In the spectra, we can also see broad solid-state features from the dust grains. The simultaneous presence of PAH emission features (C-rich) in the 6-14 $\mu \mathrm{m}$ range (Fig. 2 and the right panels of Figs. B.1, B.2, and B.3) and prominent crystalline silicate emission features (O-rich) beyond $20 \mu \mathrm{m}$ (Fig. 1 and the left panels of Figs. B.1, B.2 and B.3) illustrates the dual-dust chemistry in all 21 GB PNe. The mixed chemistry is seen not only in late-type, but also in all observed early ${ }^{4}$-type WCPNe. However, the most striking result is that dual-dust chemistry was detected in all observed wels as well as in some other GB PNe with CSs, which are very likely of none of the above types as inferred from their optical spectra (see Table 1). Spectral type of H 1-62 is unknown. The class of GB PNe with neither [WC] nor wels CSs is investigated in a subsequent paper (Górny et al. 2009, in preparation).

A broad, dust feature around $10 \mu \mathrm{m}$ is seen in a few PNe. This feature appears to be due to amorphous silicates and is seen only in 4 (Th 3-4, M 3-38, M 3-8 and H 1-40 - see Figs. B.2 and B.3) out of the $21 \mathrm{~GB}$ PNe with dual-dust chemistry. The only other known case of WCPN with such feature is SwSt 1 (see e.g. Szczerba et al. 2001). By interpreting the strength of the $11.3 \mu \mathrm{m}$ PAH band as a measure of the PAH abundance, we see

\footnotetext{
${ }^{4}$ Note that Cn 1-5 was previously observed with ISO, although the spectrum was of bad quality and the presence of dual-dust chemistry was uncertain in this early-type WCPN.
}

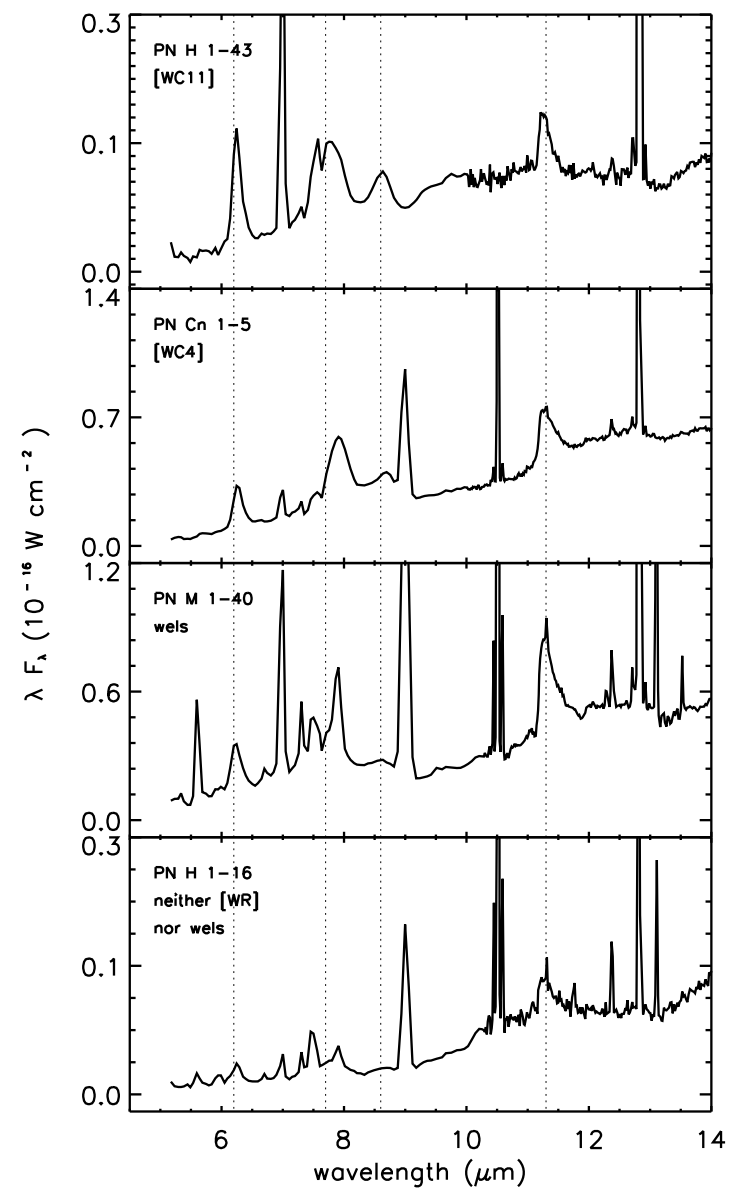

Fig. 2. Same spectra as in Fig. 1, but for wavelenghts $<14 \mu \mathrm{m}$. The positions of typical PAH features at 6.2, "7.7", 8.6 and $11.3 \mu \mathrm{m}$ are indicated by dotted vertical lines.

that these 4 sources (except M3-38) have the lowest PAH content (detection of PAHs is even tentative in the case of M3-8 see Table 1 and Fig B.3). Surprisingly, this $10 \mu \mathrm{m}$ band is seen in most non dual-dust chemistry GB sources in our sample, except H 1-12, for which we have only SH observations and we cannot deduce the presence of this band, and M 3-13 whose spectrum is of rather low quality (see Fig. B.4). These sources show only weak crystalline silicate features. The sources with $10 \mu \mathrm{m}$ dust feature are discussed in more detail by Górny et al. (2009, in preparation).

There are 14 additional $\mathrm{PNe}$ in our sample, which are not counted as GB sources. The relevant information for these sources is available only online and is collected in Table A.2 with spectra shown in Figs. B.5 and B.5. Among them, there are 6 PNe with dual-dust chemistry (3 early-[WC]; 2 wels and one with unknown type CS). Surprisingly, there is one wels ${ }^{5}$ (M 1-20) that does not display the signs of O-rich dust, but only PAHs and a broad plateau emission between 11 and $14 \mu \mathrm{m}$ (see Fig. B.5), attributed to primordial C-rich material, also observed in proto-PNe (e.g. Kwok et al. 2001). This PN, and another Crich source (Tc 1) showing unusual, hydrogenated PAHs (see Fig. B.6) will be discussed in more detail in a forthcoming paper. We note the exceptional source GLMP 698 (with CS classified as [WC8]), for which only weak bands of crystalline silicates are detected (similar to ISO target K 2-16 with a CS classified

\footnotetext{
5 The three wels $\mathrm{PNe}$ are located only slightly further than $10^{\circ}$ from the Galactic Centre, so they may as well belong to the GB population.
} 
as [WC 11]). Another 5 sources show only silicates, with no indication of C-rich dust. Out of the 14 non-GB PNe, there are 5 sources showing both, amorphous and crystalline silicates.

\section{Discussion}

The origin of the dual-dust chemistry in PNe is still unclear. In general, several mechanisms may play a role:

i) a very late thermal pulse at the end of the AGB phase (or just after), which may turn an O-rich outflow into a C-rich one (Waters et al. 1998a). Due to the enhanced mass loss, high temperature annealing may lead to the crystallization of amorphous silicates (e.g. Sylvester et al. 1999), while PAHs form from the newly released C-rich gas;

ii) evaporation of Oort-like cloud comets may release crystalline silicates (Cohen et al. 1999), while PAHs form after transformation of the star into C-rich one;

iii) the central star of the PN may exist in a binary system with a circumbinary O-rich disk that was formed long before the PN. Low temperature crystallization may take place in such a disk (Molster et al. 1999). PAHs would form after transformation of the O-rich star into a C-rich one;

iv) a low-mass main sequence star, brown dwarf, or planet may spiral into the AGB star, inducing a chemistry change as a consequence of extra-mixing (De Marco \& Soker 2002). High temperature annealing of amorphous silicates and PAH formation from the newly released $\mathrm{C}$-rich gas could be invoked;

v) for intermediate-mass stars, the strong mass loss may induce a sudden deactivation of the hot bottom burning (see e.g. García-Hernández et al. 2006) just at the end of the AGB evolution. The high temperature annealing may lead to crystallization of amorphous silicates, while carbon, again allowed to be dredged-up to the surface, may be used for the PAH formation.

In our sample of GB PNe, we see an overwhelming presence of crystalline silicates (see also Gutenkunst et al. 2008). This might be explained by the existence of long-lived O-rich disk (primordial or circumbinary) around CSs of PNe as proposed in scenarios ii) and iii). However, these explanations appear to be inconsistent with the existing observations of AGB stars in the GB, which in general do not show any evidence of crystalline silicates (Vanhollebeke 2007). In addition, Miszalski et al. (2008) reported that out of 300 GB PNe only some dozen (about $10-15 \%)$ display signatures of periodic variability (interpreted as due to binarity) in the OGLE-III data. The lack of evidence for a dominant binarity also excludes more exotic mechanisms, such as these proposed in scenario iv). Scenario v) is also not applicable to the GB PNe, since the current AGB population in the Galactic Bulge consists of mostly low-mass stars (see e.g. Uttenthaler et al. 2007, and references therein).

To elucidate the validity of the scenario i), it is important to take into account the higher (on average) metallicity of the GB population compared with the rest of Galaxy (Chiappini et al. 2009). Low-mass O-rich AGB stars in the GB, with limited efficiency in the third dredge-up, do not succeed in bringing enough carbon into the envelope to produce C-rich AGB stars in a highmetallicity environment. Hence, it seems very likely that the formation of C-rich PNe (so numerous in the GB) must originate in a final He-shell instabillity (probably violent), which exposes the $\mathrm{He}$ - and C-rich layers only when the AGB phase is terminated.

The large mass loss expected in GB PNe at the end of the AGB due to the higher metallicity compared with the average
Galactic metallicity (and thus higher amount of dust) may facilitate the mixing/removal of the remaining hydrogen from the stellar envelope leading to the formation of [WC] CSs. In addition, this violent process may be connected to the operation of strong shocks, which can increase the temperature in circumstellar shells leading to crystallization of silicates. Therefore, we suggest that process i) proposed above appear to be the most plausible scenario in explaining the formation of $\mathrm{C}$-rich $\mathrm{PNe}$ with mixed chemistry in the GB.

The detection of dual-dust chemistry in the majority of the GB PNe constitutes the first evidence that this phenomenon is not restricted to late-type WCPNe, as previously suggested. We suggest that the dual-dust chemistry in the GB PNe is related to the low mass and high metallicity of their precursors. The missing population of $\mathrm{C}$-rich AGB stars in the GB and the overall lack of crystalline silicates around GB AGB stars, suggest that the formation of PNe with dual-chemistry may be due to a final He-shell instability that leads to a simultaneous change in chemistry (PAH formation) and crystallization of pre-existing silicates. However, other possibilities for dual-dust chemistry cannot be excluded in other than GB environment. The problem clearly deserves further theoretical analysis and needs to take into account the new observational constraints provided here.

Acknowledgements. We acknowledge support from the Faculty of the European Space Astronomy Centre (ESAC) and from the Comunidad de Madrid PRICIT project S-0505/ESP-0237 (ASTROCAM). R.Sz acknowledges support from grants N203 393334 and N203 066133 . We thank to Slawek Górny for his useful comments and help during the preparation of this manuscript. We are also grateful to the referee, Dr. M. Busso, whose comments allowed us to improve the manuscript.

\section{References}

Acker, A., Marcout, J., Ochsenbein, F., Stenholm, B., \& Tylenda, R. 1992, Strasbourg - ESO catalogue of galactic planetary nebulae. Part 1; Part 2 (Garching: European Southern Observatory)

Allamandola, L. J., Tielens, A. G. G. M., \& Barker, J. R. 1985, ApJ, 290, L25 Chiappini, C., Górny, S. K.. Stasińska, G., \& Barbuy, B. 2009, A\&A, 494, 591 Cohen, M., Barlow, M. J., Sylvester, R. J., et al. 1999, ApJ, 513, L135

Cohen, M., Barlow, M. J., Liu, X.-W., \& Jones, A. F. 2002, MNRAS, 332, 879 De Marco, O., \& Soker, N. 2002, PASP, 114, 602

Egan, M. P., Price, S. D., Kraemer, K. E., et al. 2003, AFRL-VS-TR-2003-1589 García-Hernández, D. A., Abia, C., Manchado, A., \& García-Lario, P. 2006, A\&A, 452, 1049

Górny, S. K., Stasińska, G., Szczerba, R., \& Tylenda, R. 2001, A\&A, 377, 1007 Górny, S. K., Stasińska, G., Escudero, A. V., \& Costa, R. D. D. 2004, A\&A, 427, 231

Górny, S. K., Chiappini, C., Stasińska, G., \& Cuisinier, F. 2008, A\&A, submitted Gutenkunst, S., Bernard-Salas, J., Pottasch, S. R., Sloan, G. C., \& Houck, J. R. 2008 ApJ, 680, 1206

Higdon, S. J. U., Devost,D., Higdon, J. L., et al. 2004, PASP, 116, 975

Houck, J. R., Roellig, T. L., van Cleve, J., et al. 2004, ApJS, 154, 18

Kwok, S., Volk, K., \& Bernath, P. 2001, ApJ, 554, L87

Leger, A., \& Puget, J. L. 1984, A\&A, 137, L5

Miszalski, B., Acker, A., Moffat, A. F. J., Parker, Q. A., \& Udalski, A. 2008, A\&A, 488, L79

Molster, F. J., Yamamura, I., Waters, L. B. F. M., et al. 1999, Nature, 401, 563 Molster, F. J.,Waters, L. B. F. M., \& Tielens, A. G. G. M. 2002, A\&A, 382, 222 Parker, Q. A., \& Morgan, D. H. 2003, MNRAS, 341, 961

Sylvester, R. J., Kemper, F., Barlow, M. J., et al. 1999, A\&A, 352, 587

Szczerba, R., Górny, S. K., Stasińska, G., Siódmiak, N., \& Tylenda, R. 2001, Ap\&SS, 275,113

Tylenda, R., Acker, A., \& Stenholm, B. 1993, A\&AS, 102, 595

Uttenthaler, S., Hron, J., Lebzelter, T., Busso, M., Schultheis, M., \& Käufl , H. U. 2007, A\&A, 463, 251

Vanhollebeke, E. 2007, Ph.D. Thesis, KUL, Lueven

Wang, W., \& Liu, X.-W. 2007, MNRAS, 381, 669

Waters, L. B. F. M., Beintema, D. A., Zijlstra, A. A., et al. 1998a, A\&A, 331, L61

Waters, L. B. F. M., Cami, J., de Jong, T., et al. 1998b, Nature, 391, 868

Werner, M. W., Roellig, T. L., Low, F. J., et al. 2004, ApJS, 154, 1 


\section{Appendix A: Tables}

Table A.1. The other Galactic Bulge PNe observed with Spitzer/IRS (program \#3633).

\begin{tabular}{|c|c|c|c|c|c|c|c|}
\hline IRAS name & PN name & $\begin{array}{c}\text { RA } \\
\text { J2000.0 }\end{array}$ & $\begin{array}{c}\text { Dec } \\
\text { J2000.0 }\end{array}$ & Spectral type ${ }^{a}$ & $\begin{array}{c}\text { Optical diameter }{ }^{e} \\
(\operatorname{arcsec})\end{array}$ & $\begin{array}{c}\text { Distance from GC } \\
\left({ }^{\circ}\right)\end{array}$ & Dust type \\
\hline $17230-3459$ & H 1-12 & 172624.3 & -350141 & unknown & 6.8 & 7.33 & $\mathrm{O}$ \\
\hline $17385-2211$ & M 3-13 & 174136.6 & -221303 & none $^{b}$ & 0.0 & 6.78 & $\mathrm{O}$ \\
\hline $17427-3402$ & H 1-32 & 174606.3 & -340345 & none $e^{b}$ & $<5.0$ & 5.13 & $\mathrm{O}$ \\
\hline $17459-3421$ & H $1-35$ & 174914.0 & -342252 & none $e^{c}$ & 2.0 & 5.50 & $\mathrm{O}$ \\
\hline $17585-2825$ & M 2-23 & 180142.7 & -282544 & none ${ }^{d}$ & 8.5 & 3.56 & $\mathrm{O}$ \\
\hline
\end{tabular}

${ }^{a}$ None - neither [WC] nor wels; ${ }^{b}$ Górny et al. (2004); ${ }^{c}$ Wang \& Liu (2007); ${ }^{d}$ Górny et al. (2008); ${ }^{e}$ Strasbourg-ESO Catalogue of Galactic Planetary Nebulae Acker et al. (1992), $0.0-\mathrm{PN}$ is a point-like source.

Table A.2. The rest of PNe (non-Galactic Bulge) observed within Spitzer/IRS program \#3633.

\begin{tabular}{|c|c|c|c|c|c|c|c|}
\hline IRAS name & PN name & $\begin{array}{c}\text { RA } \\
\text { J2000.0 }\end{array}$ & $\begin{array}{c}\text { Dec } \\
\text { J2000.0 }\end{array}$ & Spectral type $^{a}$ & $\begin{array}{c}\text { Optical diameter }{ }^{f} \\
(\operatorname{arcsec})\end{array}$ & $\begin{array}{c}\text { Distance from GC } \\
\left({ }^{\circ}\right)\end{array}$ & Dust type \\
\hline 17597-1442 & GLMP 698 & 180238.3 & -144203 & {$[\mathrm{WC} 8]^{b}$} & - & 14.77 & $\mathrm{O}$ \\
\hline 18307-1109 & M 1-51 & 183329.2 & -110726 & {$[\mathrm{WC} 4-6]^{c}$} & 9.5 & 21.03 & mixed \\
\hline $17534-1628$ & M 1-32 & 175620.3 & -162908 & {$[\mathrm{WC} 4]^{d}$} & 7.6 & 12.70 & mixed \\
\hline $18408-1347$ & M 1-60 & 184338.4 & -134456 & {$[\mathrm{WC} 4]^{d}$} & $<10.0$ & 20.30 & mixed \\
\hline $17260-1913$ & M 1-20 & 172857.6 & -191553 & wels ${ }^{e}$ & $<7.0$ & 10.40 & $\mathrm{C}$ \\
\hline $18295-2510$ & NGC 6644 & 183234.7 & -250744 & wels ${ }^{d}$ & 2.6 & 11.14 & mixed \\
\hline $18425-3323$ & IC 4776 & 184550.7 & -332034 & wels ${ }^{d}$ & 7.5 & 13.60 & mixed \\
\hline $17561-1532$ & M1-33 & 175859.2 & -153220 & unknown & 0.0 & 13.76 & mixed \\
\hline $17119-5926$ & Hen 3-1357 & 171621.1 & -592923 & unknown & - & 31.16 & $\mathrm{O}$ \\
\hline $17225-4408$ & Cn $1-3$ & 172612.3 & -441126 & unknown & $<5.0$ & 15.75 & $\mathrm{O}$ \\
\hline $17360-1815$ & Hen 2-260 & 173857.4 & -181736 & none $^{d}$ & $<10$ & 10.77 & $\mathrm{O}$ \\
\hline $17358-2854$ & 19W32 & 173902.9 & -285635 & unknown & 24.0 & 1.44 & $\mathrm{O}$ \\
\hline $18308-2241$ & IC 4732 & 183354.6 & -223840 & unknown & 4.0 & 12.57 & $\mathrm{O}$ \\
\hline $17418-4604$ & Tc 1 & 174535.3 & -460523 & unknown & 9.6 & 17.20 & $\mathrm{C}$ \\
\hline
\end{tabular}

${ }^{a}$ None - neither [WC] nor wels; ${ }^{b}$ Parker \& Morgan (2003); ${ }^{c}$ Tylenda et al. (1993), Górny et al. (2001); ${ }^{d}$ Górny et al. (2004); ${ }^{e}$ Górny et al. (2008); ${ }^{f}$ Strasbourg-ESO Catalogue of Galactic Planetary Nebulae (Acker et al. 1992), $0.0-$ PN is a point-like source, - PN is not listed in the catalogue. 
J. V. Perea-Calderón et al.: Mixed chemistry in GB PNe, Online Material $p 2$

Appendix B: Spectra
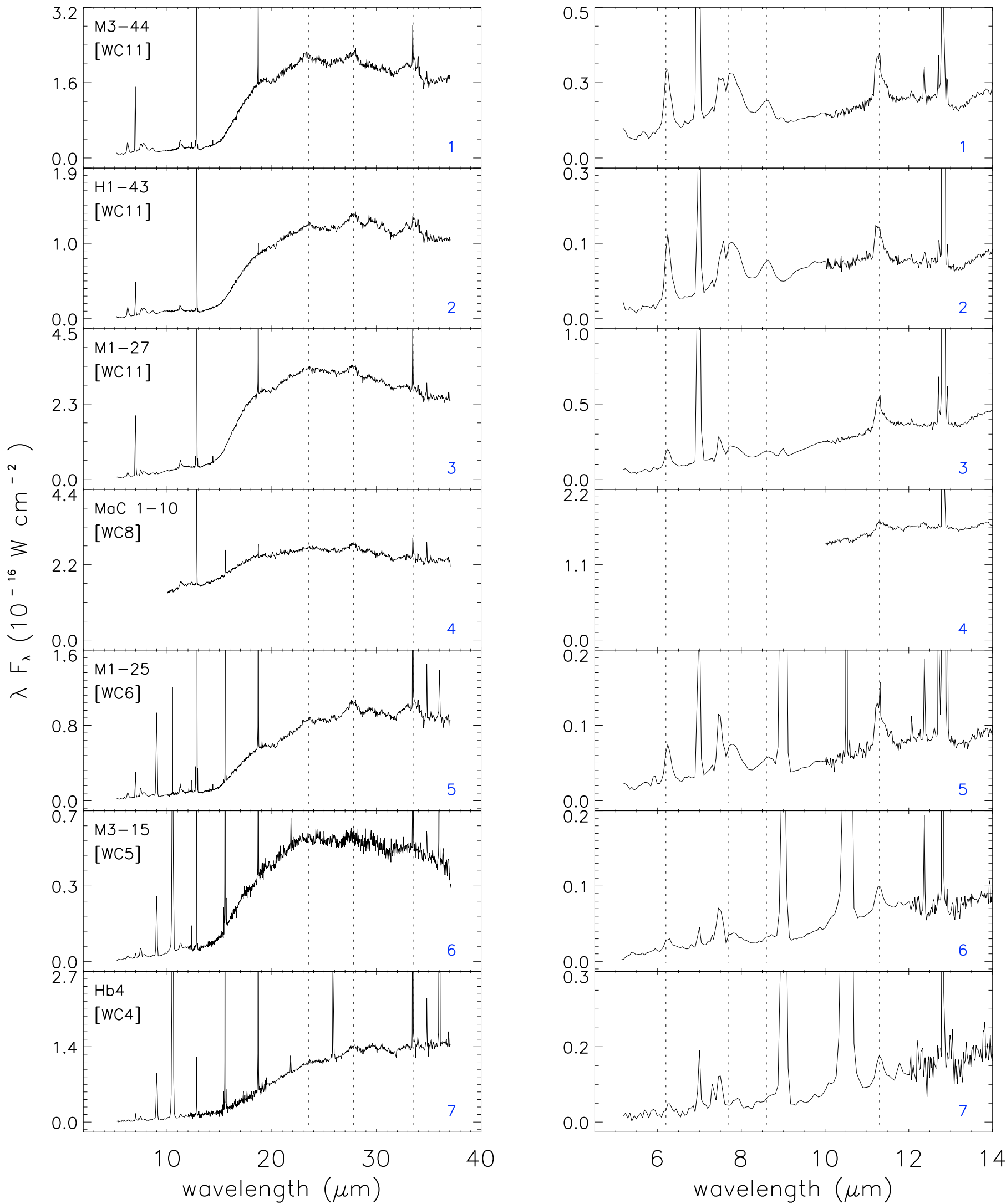

Fig. B.1. Spitzer/IRS spectra of Galactic Bulge PNe, which show dual-dust chemistry (see Table 1). The spectral type is shown under name of PN. Note the simultaneous detection of PAH emission features in the 6-14 $\mu \mathrm{m}$ range (dashed vertical lines at 6.2, "7.7", 8.6 and 11.3 $\mu \mathrm{m}-$ right panels) together with crystalline silicate features (dashed vertical lines at 23.5, 27.5 and $33.8 \mu \mathrm{m}-$ left panels). 
J. V. Perea-Calderón et al.: Mixed chemistry in GB PNe, Online Material $p 3$
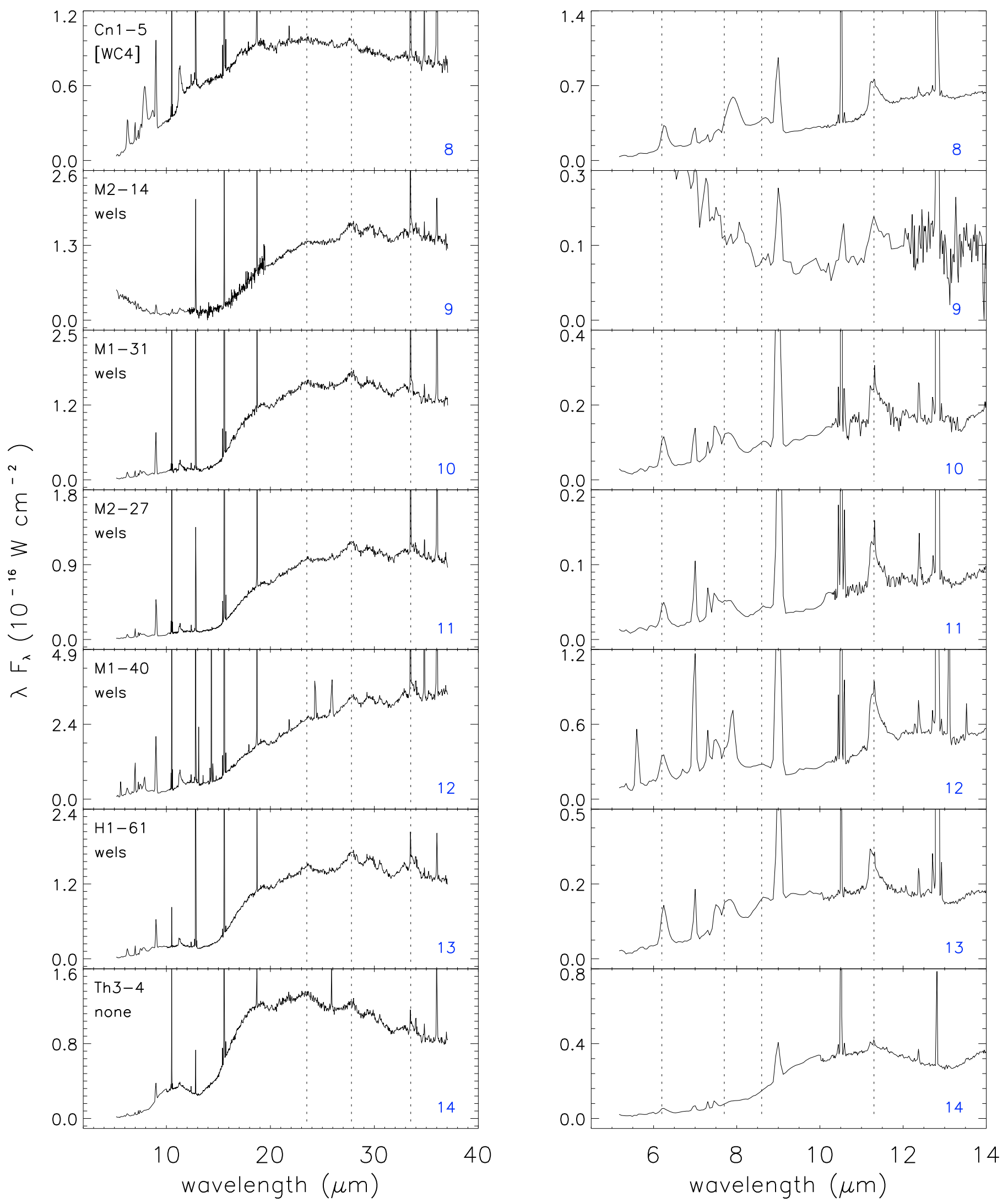

Fig. B.2. Spitzer/IRS spectra of Galactic Bulge PNe, which show dual-dust chemistry cont. (see Table 1). The spectral type is shown under name of PN; none - means neither [WC] nor wels. Note the simultaneous detection of PAH emission features in the 6-14 $\mu \mathrm{m}$ range (dashed vertical lines at 6.2, "7.7", 8.6 and $11.3 \mu \mathrm{m}$ - right panels) together with crystalline silicate features (dashed vertical lines at 23.5, 27.5 and $33.8 \mu \mathrm{m}-$ left panels). 
J. V. Perea-Calderón et al.: Mixed chemistry in GB PNe, Online Material $p 4$
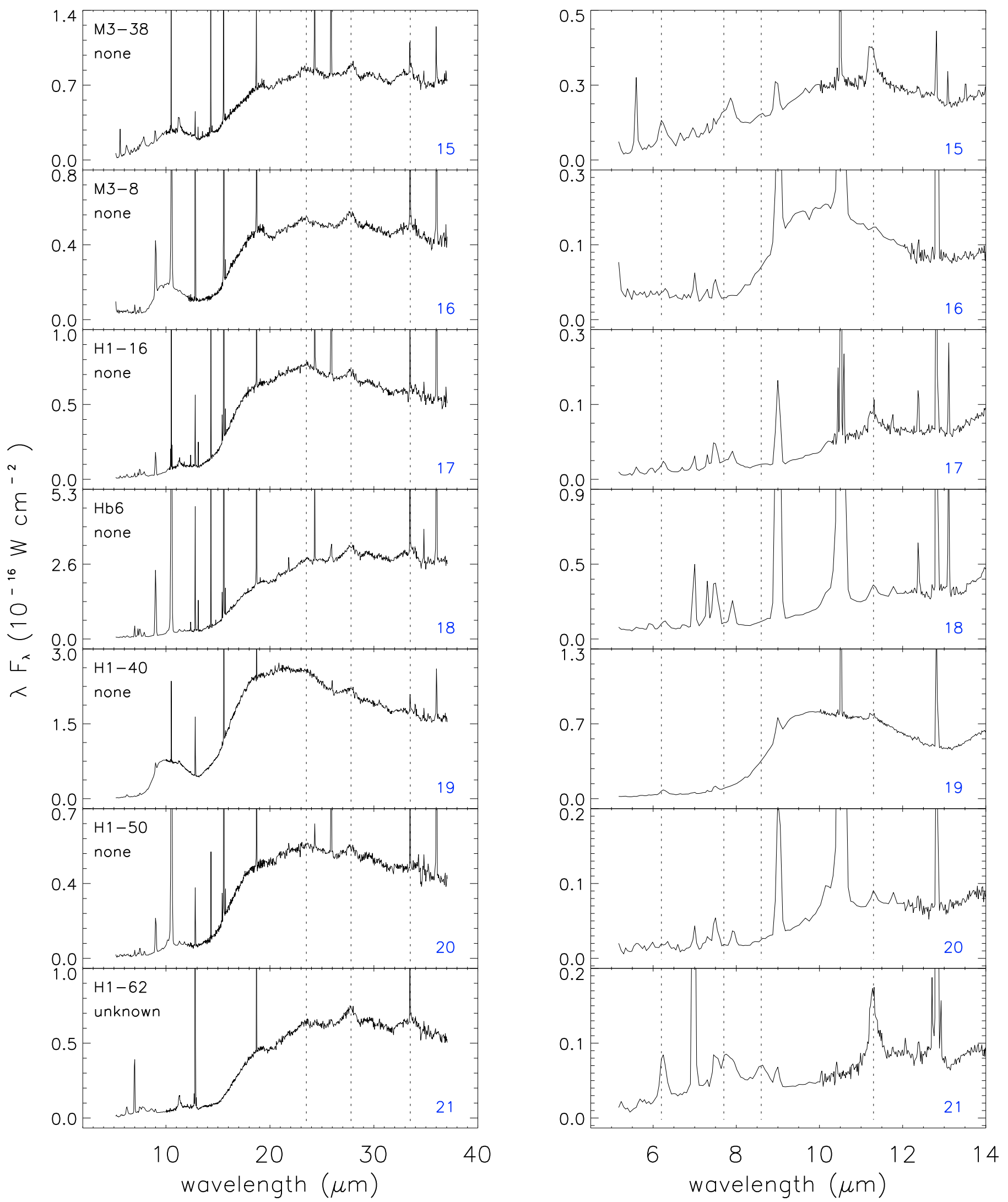

Fig. B.3. Spitzer/IRS spectra of Galactic Bulge PNe, which show dual-dust chemistry cont. (see Table 1). The spectral type is shown under name of PN; none - means neither [WC] nor wels. Note the simultaneous detection of PAH emission features in the 6-14 $\mu \mathrm{m}$ range (dashed vertical lines at 6.2, "7.7", 8.6 and $11.3 \mu \mathrm{m}$ - right panels) together with crystalline silicate features (dashed vertical lines at 23.5, 27.5 and $33.8 \mu \mathrm{m}-$ left panels). 
J. V. Perea-Calderón et al.: Mixed chemistry in GB PNe, Online Material p 5
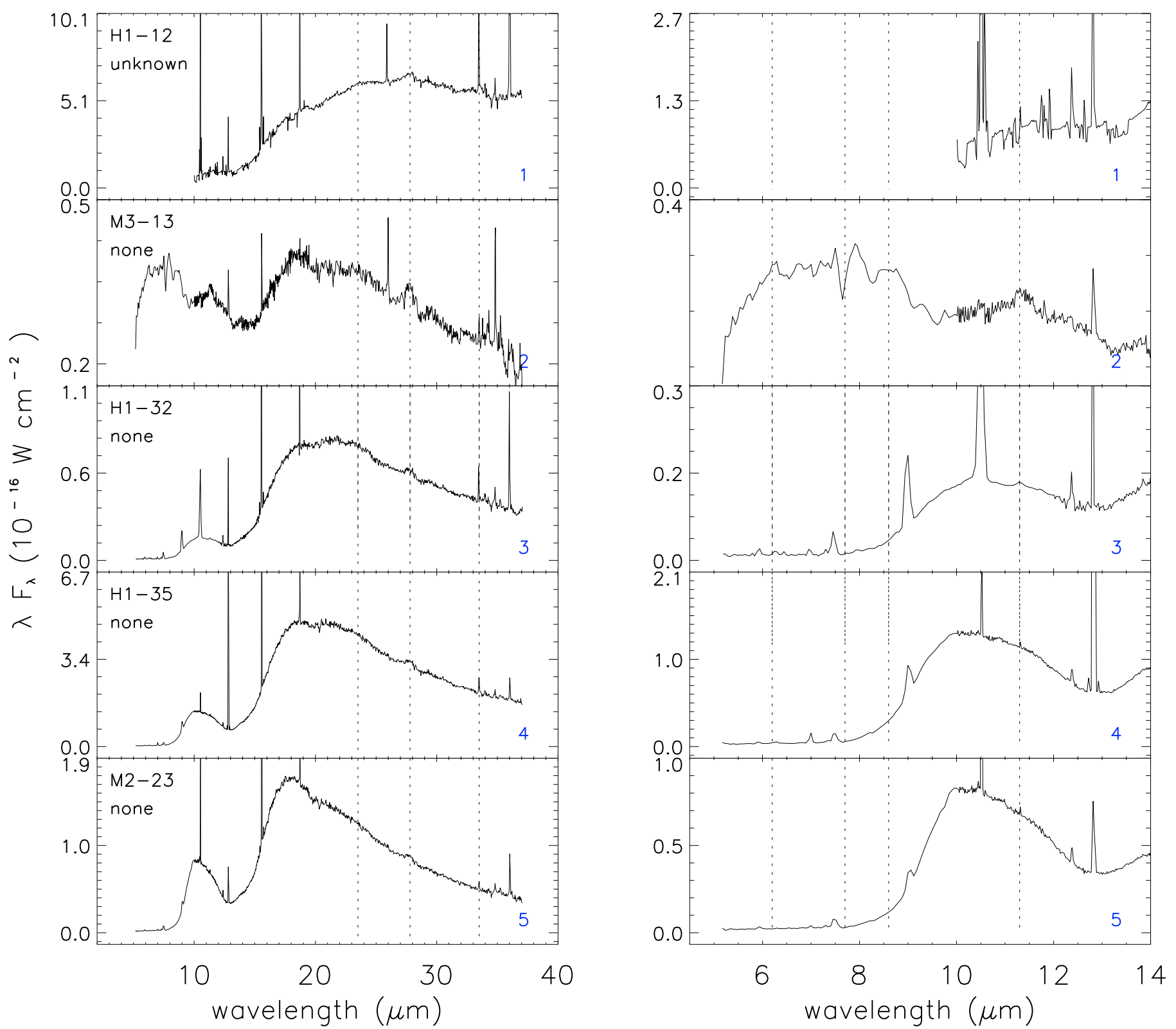

Fig. B.4. Spitzer/IRS spectra of Galactic Bulge PNe, which do not show dual-dust chemistry (see Table A.1). The spectral type is shown under name of PN; none - means neither [WC] nor wels. Dashed vertical lines at 23.5, 27.5 and $33.8 \mu \mathrm{m}$ on the left panels mark position of crystalline silicate bands, while those at 6.2, "7.7", 8.6 and $11.3 \mu \mathrm{m}$ on the right panels show position of PAH features. 
J. V. Perea-Calderón et al.: Mixed chemistry in GB PNe, Online Material $p 6$
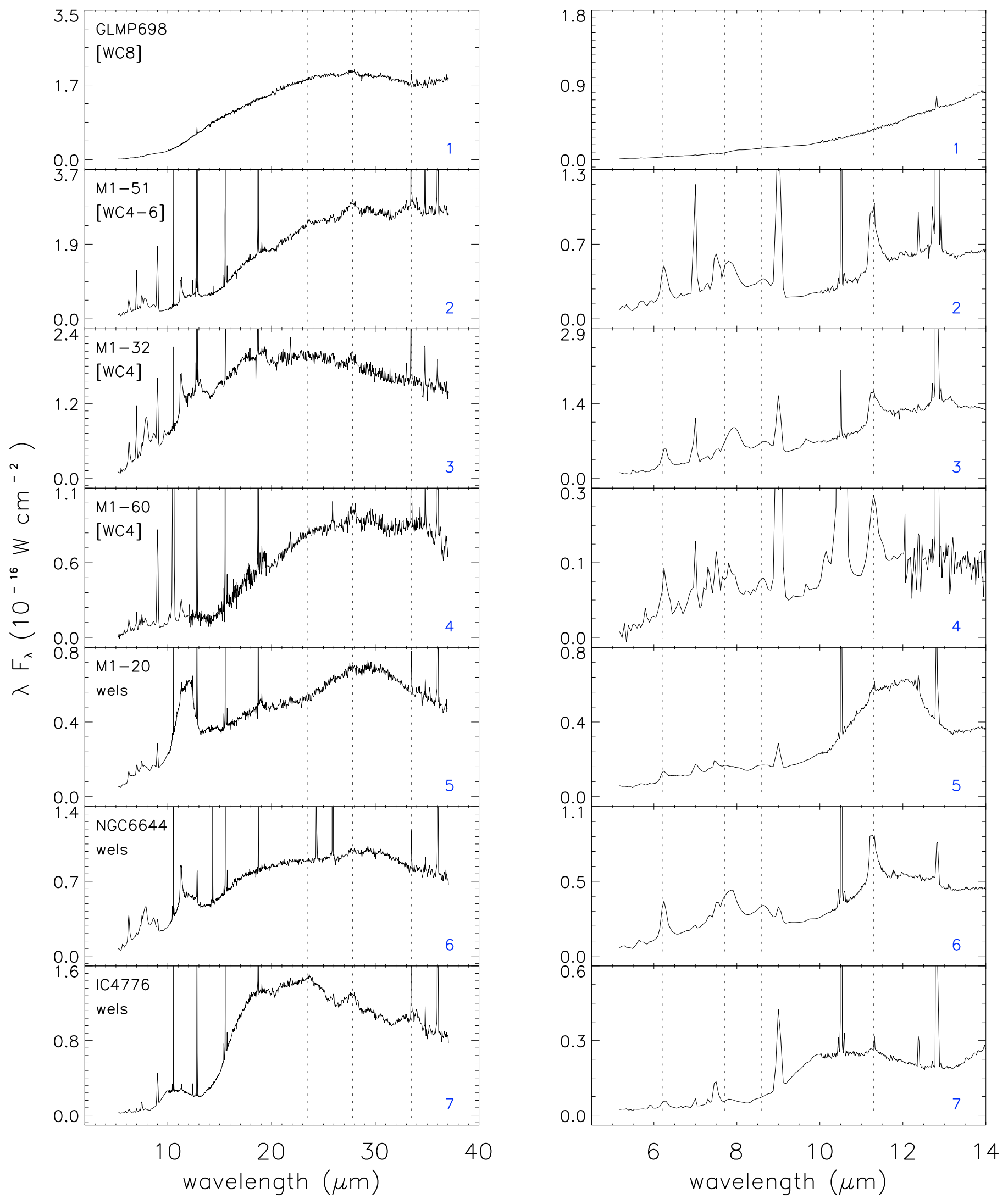

Fig. B.5. Spitzer/IRS spectra of PNe, which are classified here as non-Galactic Bulge sources (see Table A.2). The spectral type is shown under name of PN. Dashed vertical lines at 23.5, 27.5 and $33.8 \mu \mathrm{m}$ on the left panels mark position of crystalline silicate bands, while those at 6.2, "7.7", 8.6 and $11.3 \mu \mathrm{m}$ on the right panels show position of PAH features. 
J. V. Perea-Calderón et al.: Mixed chemistry in GB PNe, Online Material p 7
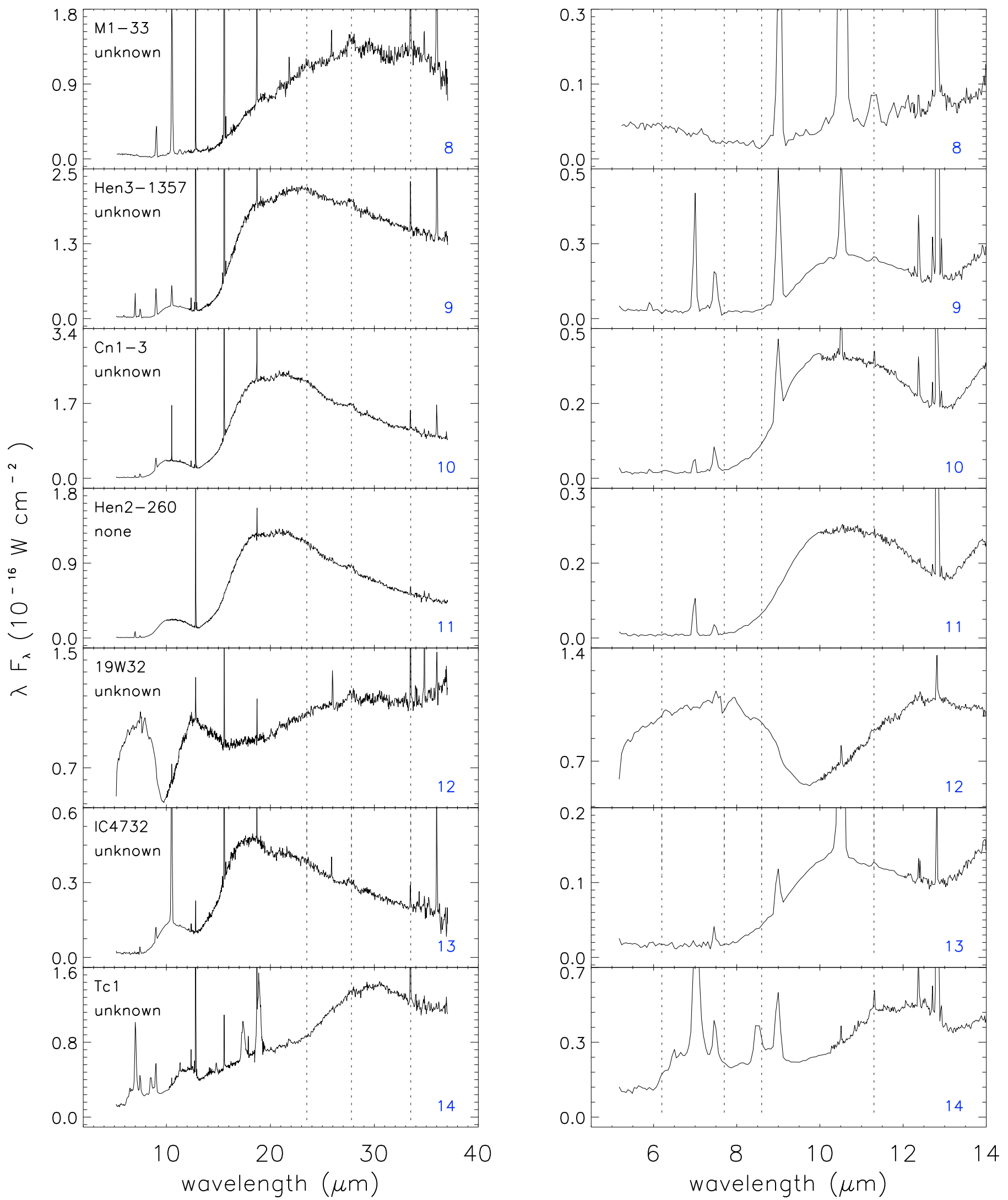

Fig. B.6. Spitzer/IRS spectra of PNe, which are classified here as non-Galactic Bulge sources cont. (see Table A.2). The spectral type is shown under name of PN; none - means neither [WC] nor wels. Dashed vertical lines at 23.5, 27.5, and $33.8 \mu \mathrm{m}$ on the left panels mark position of crystalline silicate bands, while those at 6.2, "7.7", 8.6, and $11.3 \mu \mathrm{m}$ on the right panels show position of PAH features. 